\title{
Nivel de creencias de los docentes acerca de la reforma de la educación matemática en Costa Rica ${ }^{1}$
}

\author{
Dra. Evelyn Agüero-Calvo², Instituto Tecnológico de Costa Rica, Costa \\ Rica; Dr. Luis Gerardo Meza-Cascante ${ }^{3}$, Instituto Tecnológico de \\ Costa Rica, Costa Rica y Dra. Zuleyka Suárez-Valdés-Ayala ${ }^{4}$, Instituto \\ Tecnológico de Costa Rica, Costa Rica. \\ Recibido: $\quad 26$ de febrero, 2018. \\ Aceptado: $\quad 23$ de setiembre, 2018.
}

\section{Resumen}

En este artículo se exponen los resultados de un estudio acerca del nivel de creencias que manifiestan docentes de matemática con respecto a la reforma de la educación matemática en Costa Rica, que inició en el año 2013, partiendo de la premisa de que las actuaciones de los profesores responden a esas creencias más que a las directrices oficiales o los planteamientos teóricos.

Para tal efecto se utilizó el instrumento denominado Medición de las creencias de los docentes sobre los nuevos programas de estudio de matemáticas en secundaria, construido y validado por Monge y Reyes (2017). Se utilizó una muestra de 361 docentes de colegios públicos diurnos oficiales del Ministerio de Educación Pública de Costa Rica en el año 2017. Además, se indagó la existencia de diferencias por sexo, categoría profesional y años de antigüedad.

Los resultados indican que $57 \%$ de los docentes tienen niveles altos de creencias sobre la reforma y que no se detectan diferencias en el nivel de creencias por sexo, categoría profesional o antigüedad. Asimismo, se encuentra que 65,9\% de los profesores considera que la resolución de problemas es la metodología más adecuada para abordar los temas de los nuevos programas de matemáticas. Contrariamente, un $70,4 \%$ piensa que lo propuesto en los nuevos programas de matemáticas no es factible de ser desarrollado en las aulas del sistema educativo costarricense.

1 Los autores agradecen el apoyo de la Vicerrectoría de Investigación y Extensión del Instituto Tecnológico de Costa Rica y a los docentes participantes en este proyecto.

2 Doctora en Intervención Educativa por la Universidad de Valencia (España), Máster en Matemática Educativa por el CINVESTAV de México y Bachiller en la Enseñanza de la Matemática asistida por Computadora por el Instituto Tecnológico de Costa Rica. Actualmente se desempeña como profesora catedrática e investigadora consolidada 1 en el Instituto Tecnológico de Costa Rica. Contacto: evaguero@itcr.ac.cr.

3 Doctor en Educación por la Universidad Estatal a Distancia, Máster en Administración de Empresas por el Instituto Tecnológico de Costa Rica y Licenciado en la Enseñanza de la Matemática por la Universidad Nacional. Es profesor catedrático e investigador consolidado 1 en el Instituto Tecnológico de Costa Rica (TEC). Contacto: gemeza@itcr.ac.cr.

4 Doctora en Educación por la Universidad Estatal a Distancia, Máster en Educación y Licenciada en la Enseñanza de la Matemática por la Universidad Nacional. Labora como profesora asociada e investigadora consolidada 1 en la Escuela de Matemática del Instituto Tecnológico de Costa Rica. Contacto: zsuarez@itcr.ac.cr.
Evelyn Agüero-Calvo, Luis Gerardo Meza Cascante y Zuleyka Suárez-Valdés Ayala. Nivel de creencias de los docentes acerca de la reforma de la educación matemática en Costa Rica. Revista Comunicación. Año 39, volumen 27, número 2, julio-diciembre, 2018. Instituto Tecnológico de Costa Rica. ISSN: 0379-3974 / e-ISSN1659-3820.

\section{PALABRAS CLAVE:}

Matemática, creencias, enseñanza secundaria, profesor.

\section{KEY WORDS:}

Mathematics, beliefs, secondary education, teacher. 


\section{Abstract \\ TEACHERS' BELIEF LEVEL ABOUT THE REFORM IN MATHEMATICS EDUCATION IN COSTA RICA}

This article presents the results of a study on the level of beliefs that mathematics teachers manifest regarding reforms in mathematics education in Costa Rica started in 2013, based on the premise that the teachers' actions respond to these levels of beliefs more than to official guidelines or theoretical approaches.

For this purpose, an instrument called Measurement of teachers' beliefs regarding the new mathematics study programs in secondary school designed, utilized and validated by Monge and Reyes (2017). A sample of 361 public day school teachers of the Ministry of Public Education of Costa Rica was used in 2017. In addition, possible differences according to sex, professional category, and years of seniority were researched.

The results indicate that $57 \%$ of teachers have high belief levels about the reform, and that no differences in belief level are detected according to gender, professional category, or seniority. In addition, the study found that $65.9 \%$ of the cases consider that problem-solving is the most appropriate methodology to address the topics in the mathematics programs, and $70.4 \%$ consider that what is proposed in the new mathematics programs is not feasible to be implemented in the classrooms of the Costa Rican education system.

\section{INTRODUCCIÓN}

En este artículo se reportan los resultados de una investigación acerca de los sistemas de creencias de los profesores de matemática de la educación media con respecto a la reforma que está en marcha, partiendo de la premisa de que las actuaciones de los docentes responden a esas creencias más que a las directrices oficiales o los planteamientos teóricos.

En mayo de 2012 el Consejo Superior de Educación de Costa Rica aprobó nuevos programas de matemática para la educación primaria y secundaria. El nuevo currículo constituye una profunda reforma de programas que habían sido aprobados en 1995, modificando otros que -en esencia- no habían cambiado desde 1964 (Ruiz, 2013). Al respecto, Monge y Reyes (2017) señalan:

no se trata en realidad de solo la aprobación de nuevos programas, como había ocurrido en ocasiones anteriores, sino que el acuerdo del Consejo Superior de Educación tiene implicaciones en otros ámbitos relevantes, especialmente en cuestiones metodológicas pues establece la resolución de problemas como la metodología principal (p. 12).

De acuerdo con el Ministerio de Educación Pública (en adelante, MEP) (2012, p. 15) los nuevos programas adoptan cinco ejes disciplinares "que atravie- san de forma transversal el plan de estudios y fortalecen el currículo":

- La resolución de problemas como estrategia metodológica principal.

- La contextualización activa como un componente pedagógico especial.

- El uso inteligente y visionario de tecnologías digitales.

- La potenciación de actitudes y creencias positivas en torno a las Matemáticas.

- El uso de la historia de las Matemáticas.

A partir de 2013, el Ministerio de Educación Pública dio inicio a la puesta en práctica de estos programas y ha desarrollado una actividad importante para capacitar a los docentes de matemática de la educación media y los de la educación primaria.

El concepto de creencia ha sido un tema común de las investigaciones en educación y -a pesar de su popularidad- no hay consenso en su significado. Borg (2001) establece que una creencia es:

una proposición que puede ser sostenida consciente o inconscientemente, es evaluativa en el sentido de que el individuo la acepta como verdadera y, por lo tanto, está impregnada de compromiso emotivo; 
además, sirve como una guía para el pensamiento y el comportamiento (p. 186).

Dentro del análisis de distintas definiciones sobre las creencias, se encuentra la de Moreno y Azcárate (2003), en la cual afirman lo siguiente:

Las creencias son conocimientos subjetivos, poco elaborados, generados a nivel particular por cada individuo para explicarse y justificar muchas de las decisiones y actuaciones personales y profesionales vividas. Las creencias no se fundamentan sobre la racionalidad, sino más bien sobre los sentimientos, las experiencias y la ausencia de conocimientos específicos del tema con el que se relacionan, lo que las hacen ser muy consistentes y duraderas para cada individuo (p. 267).

Tanto Knowles (1994) como Pajares (1992) (citados por Inguanzo-Arteaga, 2010, p. 28) concuerdan en que:

Las creencias acerca de la escuela y de la enseñanza se estructuran en las personas desde edades tan tempranas como pueden ser su misma experiencia en la escuela primaria. Esto repercute en que las creencias sean resistentes al cambio aun cuando se trate de profesores que hayan pasado por un proceso de formación pedagógica ( $p$. 28).

Cruz (2008) afirma que las creencias están referidas a la interpretación del mundo y que uno de sus aspectos más conflictivos es que no hay una clara distinción conceptual con el conocimiento. Al respecto, esta autora cita a Ortega y Gasset (1955) como uno de los primeros en querer hacer dicha distinción, señalando que: "la palabra idea sirve para designar todo aquello que aparece como resultado de la actividad intelectual, y las creencias, en cambio, no las pensamos sino que están presentes en todo lo que hacemos" (p. 142).

Díaz, Martínez, Roa y Sanhueza (2010) afirman que "las creencias profundamente enraizadas que tienen los docentes sobre la forma en que se aprende un contenido impregnarán sus actuaciones en el aula más que el método concreto que estén obligados a adoptar o el texto que utilizan" (p. 5). De esta manera, los educadores tienen sistemas de creencias sobre las cuales basan su manera de actuar, incluso en el ámbito profesional.

La importancia de conocer estas creencias radica -de acuerdo con Gil y Rico (2003)- en que, al conocerlas, se puede comprender mejor algunas de sus actitudes y posiciones. Inguanzo-Arteaga (2010) afirma que la investigación educativa ha modelado el concepto de creencia como un factor que permite ayudar a comprender la conducta, la toma de decisiones y el manejo de procesamiento de la información.

Díaz, Martínez, Roa, I. y Sanhueza (2010) sostienen que, cuando se desean realizar modificaciones en un sistema educativo, debe suponerse un cambio de las creencias de los docentes y estudiar a fondo los sistemas de estas para ayudar a potenciar los cambios que se pretenden. Al respecto, Vizcaíno, Cadalso y Manzano (2015) manifiestan que "la posibilidad de cambiar la enseñanza sin cambiar las creencias que a ésta subyacen parece difícil, pues sin conciencia de las razones que alientan la práctica docente resulta complejo modificar aspectos de la misma con el fin de mejorarla" (p. 258).

Adicionalmente, tal como expresa Rico (1997, citado por Gil y Rico, 2003), cada profesor da una respuesta personal a las cuestiones clave del currículo para su acción en el aula: tiene unos objetivos y para alcanzarlos trabaja unos contenidos con una determinada metodología y aplica unos criterios de evaluación:

Para diseñar planes de formación, proponer y desarrollar innovaciones curriculares y para implicar a los profesores en los procesos de cambio, parece imprescindible conocer sus concepciones y creencias. Las tareas profesionales y educativas tendrán mayores probabilidades de éxito si en su planificación consideran el pensamiento de los profesores (Gil y Rico, 2003, p. 187).

Establecer un cambio educativo es un proceso complejo, en el cual las creencias de los docentes sobre la enseñanza y el aprendizaje de las matemáticas 
son fundamentales para determinar el ritmo, ya que influyen sobre la calidad y el proceso de innovación. No es de extrañar que muchos profesores, cuando deben presentar el plan de estudios en sus clases, confíen más en sus propias creencias que en las tendencias actuales de la pedagogía. Este proceder tiene su propia racionalidad en la naturaleza práctica y cotidiana de la profesión docente. En general, los estudios sobre las creencias pedagógicas de los docentes revelan la complejidad de provocar un cambio educativo, lo cual podría ser la razón, en alguna medida, del fracaso de muchos intentos de reforma en el pasado (Handal y Herrington, 2003).

Borg (2001) por su parte, afirma que las creencias juegan un papel importante en muchos aspectos de la enseñanza, así como también lo hacen en la vida, ya que ayudan a las personas a dar sentido al mundo e influyen en cómo se percibe la información nueva, y si esta es aceptada. Por tanto, conocer esas creencias resulta imprescindible para ajustar los procesos de capacitación en procura de resultados positivos, ya que toda innovación pedagógica requiere el apoyo de los agentes implicados, siendo importante tener en cuenta las creencias de los profesores para que cualquier reforma sea exitosa (Handal y Herrington, 2003). Si estas creencias son compatibles con la innovación propuesta, la aceptación y el cambio se producirán; pero si los profesores tienen creencias opuestas, serán una barrera para la implantación de cualquier innovación educativa (Fullan y Stiegelbauer, 1991, citados por Moreno y Azcárate, 2003).

De esta manera, tener en cuenta las creencias de los docentes es de especial relevancia para introducir reformas al sistema educativo (Marchesi y Martín, 1989, citados por Moreno y Azcárate, 2003).

\section{MÉTODOS}

La investigación desarrollada tuvo un enfoque cuantitativo, con un alcance descriptivo.

\subsection{Participantes}

Los participantes en el estudio fueron 361 docentes de matemática de colegios públicos diurnos, oficiales del Ministerio de Educación Pública de Costa
Rica, en el año 2017, con las siguientes distribuciones:

Tabla 1

Distribución por sexo

\begin{tabular}{rcc}
\hline Sexo & Frecuencia & Porcentaje \\
\hline Masculino & 206 & 57.1 \\
Femenino & 155 & 42.9 \\
\hline
\end{tabular}

Fuente: Elaboración propia

Tabla 2

Distribución por categoría profesional

\begin{tabular}{lcc}
\hline \multicolumn{1}{c}{ Categoría } & Frecuencia & Porcentaje \\
\hline MT4, MT3 o MT2 & 67 & 18,6 \\
MT5 & 93 & 25,8 \\
MT6 & 201 & 55,7 \\
\hline
\end{tabular}

Fuente: Elaboración propia

Tabla 3

Distribución por años de experiencia laboral

\begin{tabular}{|c|c|c|}
\hline Rango & Frecuencia & Porcentaje \\
\hline $\begin{array}{l}\text { Más de } 5 \text { años y menos } \\
\text { de } 10\end{array}$ & 94 & 26 \\
\hline $\begin{array}{l}\text { Más de } 10 \text { años y menos } \\
\text { de } 15\end{array}$ & 118 & 32,7 \\
\hline Más de 15 años & 149 & 41,3 \\
\hline
\end{tabular}

\subsection{Instrumentos}

El nivel de creencias de los docentes fue medido con el instrumento denominado Medición de las creencias de los docentes sobre los nuevos programas de estudio de matemáticas en secundaria, construido y validado por Monge y Reyes (2017). Dicho instrumento contiene 20 ítems tipo Likert con cuatro opciones: totalmente de acuerdo, de acuerdo, en desacuerdo y totalmente en desacuerdo.

El instrumento fue autoadministrado y cada docente lo respondió en línea, de manera anónima y confidencial, de acuerdo con sus creencias, según lo afirmado en cada ítem. 


\subsection{Procedimiento}

El test descrito (en el Anexo) se aplicó a un grupo de docentes de matemática mediante una encuesta digital creada usando formularios de Google, seleccionados a partir de un listado de correos electrónicos obtenidos con la colaboración de asesores de matemática del Ministerio de Educación Pública.

\subsection{Análisis estadísticos}

Con respecto al instrumento de medición, se realizó un estudio de sus características psicométricas: índice de discriminación, confiabilidad y unidimensionalidad. Además, se clasificaron los puntajes de los autorreportes con respecto al nivel de creencias en cuatro categorías: muy baja, baja, alta y muy alta.

Para determinar la validez del instrumento se llevó a cabo un estudio de la discriminación de cada ítem calculando la correlación entre el puntaje del ítem y la suma del puntaje en los otros ítems (Lozano y De la Fuente, 2009). La interpretación de los rangos de discriminación se hizo de acuerdo con las recomendaciones de Lozano y De la Fuente-Solana (2009).

La confiabilidad del instrumento se basó en la técnica denominada Alfa de Cronbach, siguiendo a Cea (1999), la cual establece el mínimo aceptado en 0,80 .

Para determinar la unidimensionalidad del instrumento -esto es que esencialmente mida un mismo constructo- se utilizó el análisis factorial dado, que es la técnica más usada en estos casos (Jiménez y Montero, 2013), después de calcular el índice de adecuación muestral de Kayser-Meyer-Olkin (KMO) y la prueba de esfericidad de Bartlett, según lo indicado por Carmines y Zeller (1979, citados por Burga, 2006).

Como parte del análisis descriptivo de los datos, se hizo una baremación de los puntajes con el propósito de establecer una distribución de la muestra en cuatro categorías, siguiendo la interpretación propuesta por Pérez-Tyteca (2012): identificar los valores cercanos a 1 con un nivel muy bajo de creencias acerca de la reforma, valores cercanos a 2 con un nivel bajo, valores cercanos a 3 con un nivel alto y los valores cercanos a 4 con un nivel muy alto.

Posteriormente se contrastaron las siguientes tres hipótesis:

Hipótesis 1: No existen diferencias en el nivel de creencias entre hombres y mujeres.

Hipótesis 2: No existen diferencias en el nivel de creencias por categoría profesional.

Hipótesis 3: No existen diferencias en el nivel de creencias por antigüedad.

En el caso de la hipótesis 1, relacionada con la diferencia de medias en dos categorías, se utilizó la prueba paramétrica $t$ de Student, asumiendo la distribución normal de los datos y basándose en el teorema del límite central. Para las hipótesis 2 y 3 , que están relacionadas con varias categorías, se usó la prueba ANOVA después de verificar la homogeneidad de varianzas con la prueba de Levene.

El tamaño del efecto solo será calculado en aquellos casos en los que haya diferencias estadísticamente significativas, usando la $d$ de Cohen (Ripoll, 2011), la cual representa el número de desviaciones típicas que separan los dos grupos. La interpretación de la magnitud de los tamaños de los efectos medidos por la $d$ de Cohen se basa en las referencias dadas por Cohen (Ripoll, 2011): alrededor de 0,20 hay una pequeña diferencia, cerca de 0,50 una moderada diferencia y 0,80 o más una gran diferencia.

Finalmente, se muestran los resultados obtenidos de dos ítems relacionados con la pertinencia de la reforma y la viabilidad de implementarla.

\section{RESULTADOS}

\subsection{Instrumento de medición}

Para el estudio de las características psicométricas del instrumento utilizado se determinó, en primera instancia, que los índices de discriminación obtenidos para cada ítem $(\geq 0,499)$ son adecuados, por lo que no es necesario eliminar ninguno.

Adicionalmente, el valor obtenido del estadístico denominado Alfa de Cronbach fue $\alpha=0,951$, lo 
cual indica que el instrumento es altamente confiable.

Posteriormente, se determinó que el instrumento es unidimensional según el índice $\mathrm{KMO}$, cuyo valor fue de 0,972 con un valor $p<0,05$ y un primer factor que representa $52,82 \%$ de la varianza total explicada.

\subsection{Clasificación del nivel de creencias}

La media en el nivel de creencias con respecto a la reforma $(M=52,13, S D=10,41)$ fue significativamente mayor que la media de la escala $(t(360)=$ $3,888, p<0,05)$, indicando que, globalmente, los docentes presentan un nivel de creencias mayor que el promedio, es decir, manifiestan un nivel de creencias favorable.

Con respecto a los puntajes obtenidos en el nivel de creencias, la tabla 4 muestra los porcentajes por categoría:

Tabla 4

Categorías del nivel de creencias

\begin{tabular}{lcc}
\hline Categoría & Frecuencia & Porcentaje \\
\hline Muy baja & 19 & 5,3 \\
Baja & 138 & 38,2 \\
Alta & 169 & 46,8 \\
Muy alta & 35 & 9,7 \\
\hline
\end{tabular}

Fuente: Elaboración propia

Estos resultados muestran que, aproximadamente, el $57 \%$ de los docentes tiene un nivel de creencias alto o muy alto y $43 \%$ un nivel bajo o muy bajo sobre la reforma educativa.

\subsection{Contraste hipótesis 1}

Para contrastar la primera hipótesis, se comparó la media de los hombres $(M=52,84, S D=11,28)$ con la media de las mujeres $(M=51,18, S D=9,08)$ y no se encontró una diferencia estadísticamente significativa entre ellas $(t(359)=1,506, p>0,05)$. Es decir, se acepta la hipótesis nula de que no existen diferencias en el nivel de creencias entre hombres y mujeres.

\subsection{Contraste hipótesis 2}

Para contrastar la segunda hipótesis, se comparó la media de las tres categorías profesionales utilizando la prueba ANOVA, dado que la prueba de Levene $(p>0,05)$ indica que hay homogeneidad de varianzas. Se determinó que no existen diferencias estadísticamente significativas entre los valores de las medias de las tres categorías $(F(358)=2,916, p$ $>0,05)$. Se acepta entonces la hipótesis nula, la cual establece que no existen diferencias en el nivel de creencias según la categoría profesional.

\subsection{Contraste hipótesis 3}

Para contrastar la tercera hipótesis se comparó la media de los tres rangos de antigüedad utilizando la prueba ANOVA, ya que la prueba de Levene ( $p$ $>0,05$ ) indica que hay homogeneidad de varianzas. Se determinó que no existen diferencias estadísticamente significativas entre algún valor de las medias de los tres rangos $(F(358)=2,525, p>0,05)$. Por lo tanto, se acepta la hipótesis nula, la cual demuestra que no existen diferencias en el nivel de creencias según la antigüedad.

\subsection{Sobre la pertinencia de la reforma y la viabilidad de implementarla}

El ítem 1 del test indica lo siguiente: "La resolución de problemas es la metodología más adecuada para abordar los temas de los nuevos programas de matemáticas". Las respuestas de los docentes indican que el $65,9 \%$ está totalmente de acuerdo o de acuerdo (60,9\% y $5 \%$, respectivamente) con la afirmación y $34,1 \%$ totalmente en desacuerdo o en desacuerdo (3,6\% y $30,5 \%$, respectivamente).

Por su parte, el ítem 19 plantea lo siguiente: "Lo propuesto en los nuevos programas de matemáticas es factible de ser desarrollado en las aulas del sistema educativo costarricense". Las respuestas para este ítem señalan que un $70,4 \%$ de entrevistados está totalmente en desacuerdo (o en desacuerdo $(24,7 \%$ y $45,7 \%$, respectivamente) y un $29,7 \%$ está de acuerdo o totalmente de acuerdo $(26,9 \%$ y $2,8 \%$, respectivamente). 


\section{DISCUSIÓN}

La investigación se enfocó en determinar el nivel de creencias que manifiestan los docentes de matemática sobre la reforma de la educación matemática en Costa Rica. Los resultados indican que el 57\% de los profesores tienen niveles altos de creencias sobre la reforma, lo que en otros términos implica que un $47 \%$ presenta niveles bajos. Este hallazgo es relevante porque sugiere que una cantidad apreciable de los docentes de matemática, en cuyas manos está la implementación de la reforma, demuestra niveles de creencias no favorables a la reforma educativa de la que son actores de primer orden.

Desde la perspectiva práctica, el hallazgo sugiere que las autoridades del MEP deben tomar acciones para modificar esta situación, e incorporar en los procesos de formación de los docentes en servicio diversas acciones, dirigidas a cambiar las creencias negativas sobre la reforma. De lo contrario, es poco factible que se puedan lograr resultados positivos, dado que -como indican Díaz et al (2010)- las creencias arraigadas de los profesores impregnan sus actuaciones en el aula de una forma mayor que la metodología particular que estén obligados a adoptar.

Por otra parte, los resultados que prueban que no hay diferencias en el nivel de creencias por sexo, categoría profesional o antigüedad, permiten concluir que las acciones que emprenda el MEP para mejorar el nivel de creencias de los docentes sobre dicha reforma educativa, pueden enfocarse de manera general, sin que parezca necesario hacerlo de forma separada por sexo, categoría profesional o la cantidad de años de experiencia de los educadores. Este hallazgo es positivo porque simplifica el esquema que se vaya a adoptar para mejorar el nivel de las creencias.

Finalmente, es sorprendente el contraste que se observa en las respuestas del ítem 1 y el ítem 20. En efecto, se aprecia que un porcentaje alto de los docentes $(65,9 \%)$ manifiesta una creencia positiva sobre la pertinencia de la reforma. Pero a la vez, un porcentaje aún más alto (70,4\%) se expresa negativamente sobre la viabilidad de concretarla en las aulas. Aunque la investigación no es capaz de establecer las causas de esta situación por su carácter descriptivo, sí permite sugerir que las autoridades competentes deben trabajar en dicha identificación.

Las creencias negativas sobre la factibilidad de desarrollar en el aula los planteamientos de la reforma son una grave amenaza al éxito que se espera (como país), debido a que, si las creencias que tiene el docente no están alineadas con las pretensiones de los nuevos programas de matemática de reciente aprobación, su implantación exitosa se ve seriamente amenazada.

\section{CONCLUSIONES}

La investigación permite concluir lo siguiente:

- No existen diferencias en el nivel de creencias sobre la reforma matemática entre los docentes según el sexo.

- No hay diferencias en el nivel de creencias sobre la reforma matemática según la antigüedad laboral de los profesores.

- Tampoco se presentan diferencias en el nivel de creencias sobre la reforma matemática, según la categoría profesional de los educadores.

- El $43 \%$ de los docentes encuestados tiene un nivel de creencias bajo o muy bajo sobre la reforma matemática y el 57\%, un nivel de creencias alto o muy alto sobre la reforma matemática.

- Un $65,9 \%$ de los docentes encuestados considera que la resolución de problemas es la metodología más adecuada para abordar los temas de los nuevos programas de matemáticas.

- Finalmente, el 70,4\% de los profesores considera que lo propuesto en los nuevos programas de matemáticas no es factible de ser desarrollado en las aulas del sistema educativo costarricense.

\section{REFERENCIAS BIBLIOGRÁFICAS}

Alfaro, A., Alpízar M., Arroyo, J., Gamboa, R. e Hidalgo, R. (2004). Enseñanza de las matemáticas en Costa Rica: elementos para un diagnóstico. Heredia, Costa Rica: Universidad Nacional. 
Borg, M. (2001). Key concepts in ELT. Teachers' beliefs. ELT Journal, 55 (2), 186-188.

Burga, A. (2006). La unidimensionalidad de un instrumento de medición: perspectiva factorial. Revista de Psicología de la PUCP, XXIV (1), 53-80.

Carr, W. (2002). Una teoría para la educación. Hacia una investigación educativa crítica. Ediciones Morata.

Cea, M. (1999). Metodología cuantitativa: estrategias y técnicas de investigación social. Madrid, España: Síntesis.

Chaves, E. (2007). Una valoración sobre la enseñanza de la Estadística en los colegios académicos diurnos: regiones educativas de San José, Alajuela, Heredia, Pérez Zeledón y Upala (Tesis sometida a consideración del tribunal examinador del Programa de Doctorado Latinoamericano en Educación de la Universidad Estatal a Distancia). UNED, Costa Rica.

Chaves, E., Castillo, M., Chaves, E., Fonseca, J. y Loría, R. (2010). La enseñanza de las matemáticas en la secundaria costarricense: entre la realidad y la utopía. Tercer informe Estado de la Educación. Consejo Nacional de Rectores. Costa Rica.

Cruz, I. (2008). Creencias pedagógicas de profesores: el caso de la licenciatura en nutrición y ciencia de los alimentos en México. Revista Qurriculum, 21, 137-156. Recuperado de http://webpages.ull.es/users/revistaq/ANTERIORES/numero21/cruz.pdf.

Díaz, C., Martínez, P., Roa, I. y Sanhueza, G. (2010). Los docentes en la sociedad actual: sus creencias y cogniciones pedagógicas respecto al proceso didáctico. Polis, 25, 1-14. Recuperado de http:// polis.revues.org/625.

Gil, F. y Rico, L. (2003). Concepciones y creencias del profesorado de secundaria sobre enseñanza y aprendizaje de las matemáticas. Enseñanza de las Ciencias, 21(1), 27-47. Recuperado de www. raco.cat/index.php/ensenanza/article/viewFile/21885/21719.
Handal, B. y Herrington, A. (2003). Mathematics teachers' beliefs and curriculum reform. Mathematics Education Research Journal, 15 (1), 59-69.

Inguanzo-Arteaga, G. (2010). Creencias de los profesores de nivel de licenciatura sobre la naturaleza del conocimiento y los procesos de enseñanza y aprendizaje (Tesis doctoral). Universidad de Puebla, México. Disponible en https://rei.iteso. mx/bitstream/handle/11117/1206/6-Gonzalo-Inguanzo-Puebla.pdf?sequence $=2$.

Jiménez, K. y Montero, E. (2013). Aplicación del modelo de Rasch, en el análisis psicométrico de una prueba de diagnóstico en matemática. Revista digital Matemática, Educación e Internet, 13(1), $1-23$.

Knowles, J. G. (1994). Metaphors as windows on a personal history: A beginning teachers' experience. Teacher Education Quarterly, 21 (1), 37-63.

Lozano, L. y De la Fuente-Solana, E. (2009). Diseño y validación de cuestionarios. En Pantoja-Vallejo (coord.), Manual básico para la realización de tesinas, tesis y trabajos de investigación, pp. 251-274. España: Editorial EOS.

Meza, L. G., Agüero, E. y Calderón, M. (2012). La teoría en la práctica educativa: Una perspectiva desde la experiencia de docentes graduados/as de la carrera "Enseñanza de la matemática asistida por computadora". Revista digital Matemática, Educación e Internet, 13(1), 1-24. Recuperado de http://www.tec-digital.itcr.ac.cr/revistamatematica/ARTICULOS_V13_N1_2012/RevistaDigital_ Meza_V13_n1_2012/RevistaDigital_Meza_V13_ n1_2012.pdf

Ministerio de Educación Pública. (2012). Programas de estudio de matemáticas. I y II Ciclo de la Educación Primaria. III Ciclo de Educación General Básica y Educación Diversificada. San José, Costa Rica.

Monge, C. y Reyes, N. (2017). Elaboración de un instrumento de medición de las creencias de los docentes hacia los nuevos programas de estudio de matemáticas en secundaria (Tesis para optar por el grado de Licenciatura en la Enseñanza de 
la Matemática con Entornos Tecnológicos). Escuela de Matemática, Instituto Tecnológico de Costa Rica.

Moreno, M. y Azcárate, G. (2003). Concepciones y creencias de los profesores universitarios de matemáticas acerca de la enseñanza de las ecuaciones diferenciales. Enseñanza de las Ciencias, 21(2), 265-280.

Ortega y Gasset, J. (1955). Ideas y creencias. Madrid, España: Espasa - Calpe, S.A.

Pajares, M. F. (1992) Teachers' beliefs and educational research: cleaning up messy construct. Review of Educational Research, 62(3), 307 - 332.

Pérez-Tyteca, P. (2012). La ansiedad matemática como centro de un modelo causal predictivo de la elección de carreras (Tesis doctoral). Universidad de Granada, España.

Rico, L. (1997). Bases teóricas del currículo de matemáticas. Madrid: Síntesis.

Ripoll, J. (2011). La d de Cohen como tamaño del efecto. Recuperado de: http://clbe.wordpress. com/2011/10/26/la-d-de-cohen-como-tamanodel-efecto.

Ruiz, A. (2013). Reforma de la Educación Matemática en Costa Rica. Cuadernos de Investigación y Formación en Educación Matemática, Año 8, № Especial. Universidad de Costa Rica, Centro de Investigaciones Matemáticas y Metamatemáticas, San José, Costa Rica. Recuperado de http://revistas.ucr.ac.cr/index.php/cifem/article/view/11125.

Vizcaíno, A., Cadalso, A. y Manzano, M. (2015). Adaptación de un cuestionario para evaluar las creencias epistemológicas sobre la matemática de profesores de secundaria básica. Revista Complutense de Educación, 26(2), 255-273.

\section{ANEXO}

\section{Instrumento: Medición de las creencias de los docentes sobre los nuevos programas de estudio de matemáticas en secundaria}

\section{Ítems:}

1) La resolución de problemas es la metodología más adecuada para abordar los temas de los nuevos programas de matemáticas (aprobados en el 2012).

2) Los problemas contextualizados son los más apropiados para tratar los temas de los nuevos programas de matemáticas.

3) Uno de los propósitos fundamentales de los nuevos programas de matemáticas es generar un ambiente de motivación en el aula.

4) Uno de los propósitos fundamentales de los nuevos programas de matemáticas es promover un alto razonamiento matemático en las y los estudiantes.

5) Los nuevos programas de matemáticas favorecen el trabajo estudiantil independiente en el aula.

6) Los nuevos programas de matemáticas favorecen la construcción de capacidades para la manipulación de objetos matemáticos de naturaleza abstracta.

7) Los nuevos programas de matemáticas pretenden desarrollar habilidades en los y las estudiantes para enfrentarse a los retos del mundo del que forman parte.

8) Los nuevos programas de matemáticas son una excelente opción para mejorar la formación matemática de las y los estudiantes en secundaria.

9) Los nuevos programas de matemáticas inciden positivamente en el aprendizaje de las y los estudiantes.

10) La perseverancia hacia el aprendizaje de la matemática se puede desarrollar con los nuevos programas de matemáticas. 
11) Los nuevos programas de matemáticas proponen la participación activa y colaborativa del estudiante dentro del aula.

12) La metodología de resolución de problemas de los nuevos programas de matemáticas motiva a los y las estudiantes a aprender matemáticas.

13) El enfoque metodológico de los nuevos programas de matemáticas permite brindarle a la matemática un rostro más humano.

14) El enfoque metodológico de los nuevos programas de matemáticas permite que los y las estudiantes disfruten al aprender matemática.

15) Los nuevos programas de matemática fortalecen actitudes, creencias y valores positivos hacia la matemática en las y los estudiantes.

16) La incorporación de más contenidos en estadística y probabilidad en los nuevos programas de matemáticas es un cambio pertinente.

17) Los nuevos programas de matemáticas promueven el fortalecimiento del razonamiento lógico en el desarrollo de los contenidos.

18) Los nuevos programas de matemáticas fomentan el aprendizaje de métodos y estrategias para plantear y resolver problemas.

19) Lo propuesto en los nuevos programas de matemáticas es factible de ser desarrollado en las aulas del sistema educativo costarricense.

20) Los nuevos programas de matemáticas favorecen que los contenidos sean adaptados para ser enseñados mediante la resolución de problemas. 